\title{
Qual è il contributo dello studio DOPPS nel caratterizzare il concetto di dose di dialisi?
}

\author{
A. Cavalli, G. Pontoriero
}

S.C. Nefrologia e Dialisi, Ospedale “Alessandro Manzoni”, Lecco

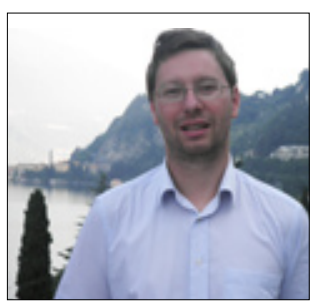

Andrea Cavalli

\section{Introduzione}

L'emodialisi è nata nel 1945 come uno strumento per trattare i casi di insufficienza renale acuta, si è poi progressivamente affermata come terapia salvavita per i pazienti affetti da insufficienza renale cronica severa, altrimenti destinati ad una prognosi infausta, e successivamente diffusa a un sempre maggiore numero di soggetti (1).

In considerazione del fatto che l'attuale schema di emodialisi più comune - costituito da 3 sedute di quattro ore alla settimana - è in grado di fornire solo una percentuale molto ridotta dell'attività omeostatica e di clearance della funzione renale nativa, sin dai primi anni della sua applicazione è emersa la necessità di definire e valutare la cosiddetta adeguatezza del trattamento emodialitico. Inizialmente essa si basava sul raggiungimento di due obiettivi fondamentali, rappresentati dalla scomparsa dei segni e sintomi secondari allo stato uremico e dalla riabilitazione del paziente (2). Un'altra definizione risalente agli anni Settanta (ma di fatto valida tutt'ora), indicativa di un approccio olistico al problema, considerava la terapia emodialitica adeguata quando permetteva la riabilitazione completa del paziente, un apporto nutrizionale soddisfacente, una produzione sufficiente di globuli rossi, il mantenimento di normali valori pressori e la prevenzione della neuropatia uremica (3). Tuttavia, queste definizioni sono limitate dal fatto di essere soggettive e di necessitare di un monitoraggio molto stretto del paziente, volto ad evitare un tardivo riscontro di uno stato di "sottodialisi”.

Per questo motivo, si è cercato di sviluppare una definizione più oggettiva di adeguatezza terapeutica che utilizzasse parametri di laboratorio per calcolare degli indici quantitativi della dose di dialisi somministrata al paziente. Tra le cosiddette "tossine uremiche", l'urea è stata scelta quale marker per valutare l'adeguatezza di dialisi attraverso modelli cinetici sviluppati da Frank Gotch e John Sargent all'inizio degli anni Settanta, sulla base della legge di conservazione di massa (4). L'urea è una molecola di piccole dimensioni, di per sé non tossica, il cui volume di distribuzione è equivalente a quello dell'acqua corporea. L'urea è considerata, appunto, il marker delle molecole di basso peso molecolare, anche se occorre ricordare l'esistenza di numerose altre "tossine uremiche", di medio ed alto peso molecolare.

Nel corso degli anni, due fondamentali studi randomizzati e controllati, il "National Cooperative Dialysis Study" (NCDS) (5) e l'HEMO Study (6), e numerosi studi osservazionali hanno cercato di stabilire criteri oggettivi per definire l'adeguatezza dialitica, i fattori che contribuiscono al suo raggiungimento e il suo significato prognostico.

Anche il Dialysis Outcomes and Practice Patterns Study (DOPPS) - studio internazionale, prospettico e osservazionale, iniziato nel 1996 con il fine di raccogliere informazioni riguardanti le correnti pratiche circa la gestione dei pazienti emodializzati - ha valutato numerosi aspetti correlati alla dose di dialisi (7).

In questa rassegna, dopo aver brevemente descritto $\mathrm{i}$ risultati ottenuti nei due importanti trials randomizzati NCDS ed HEMO Study, ci dedicheremo a esporre i dati più interessanti provenienti dall'analisi dello studio DOPPS, che, una volta di più, rappresenta l'esempio classico di come studi osservazionali caratterizzati da criteri metodologici chiari e specifici possano permettere di ottenere delle informazioni di buona qualità nell'ambito della pratica clinica quotidiana, da testare poi come ipotesi di ricerca in studi randomizzati e controllati disegnati ad hoc (8). 


\section{Dose di dialisi adeguata: un obiettivo possibile per tutti?}

Sulla base del modello cinetico dell'urea applicato in un'analisi secondaria dello studio NCDS, Gotch e Sargent hanno elaborato un indice della dose di dialisi - definito "Kt/V dell'urea" - che mette in relazione la quantità di clearance dell'urea $(\mathrm{K})$ fornita al paziente nel tempo $t$ di trattamento dialitico, con il volume di distribuzione dell'urea (V) (9).

In dialisi, l'urea segue una cinetica depurativa bicompartimentale che si manifesta con un rapido aumento, o rebound, della sua concentrazione al termine della seduta emodialitica. Se nel calcolo del $\mathrm{Kt} / \mathrm{V}$ si tiene conto di questo fenomeno di rebound post-dialitico, si parla di double pool $\mathrm{Kt} / \mathrm{V}(\mathrm{dpKt} / \mathrm{V})$ o Kt/V equilibrato $(\mathrm{eKt} / \mathrm{V})$. Quando, invece, l'effetto del rebound post-dialitico dell'urea non è tenuto in considerazione, si parla di single pool Kt/V (spKt/V). Il dpKt/V è una misura più accurata della dose di dialisi, generalmente inferiore di 0,15-0,20 rispetto al spKt/V (2).

Cosi, dopo aver definito un termine oggettivo di riferimento, il dibattito scientifico nefrologico si è spostato verso la necessità di individuare il target di dose di dialisi da raggiungere nel trattamento dei pazienti.

Alla fine degli anni Settanta, lo studio NCDS (5) è stato proprio disegnato per questo motivo, randomizzando 160 pazienti emodializzati in 4 bracci di trattamento, comprendenti due diversi tempi di durata della seduta depurativa (2,5-3,5 vs 4,5-5 ore) e due diversi livelli di TAC ("time averaged concentration") dell'urea (100 vs $50 \mathrm{mg} / \mathrm{dl}$ ). L'analisi primaria dello studio ha dimostrato l'effetto significativo di più bassi valori di TAC dell'urea nel ridurre la morbilità dei pazienti, mentre un più lungo tempo di trattamento determinava "soltanto" un trend verso migliori risultati, nei confronti dei rispettivi gruppi di confronto. È stata però la già citata analisi secondaria di Gotch e Sargent che ha mostrato come mortalità, ospedalizzazione e sintomi uremici fossero strettamente correlati ai valori di Kt/V dell'urea e sostanzialmente più elevati in presenza di un Kt/V dell'urea inferiore a 0,8. Pertanto, un Kt/V dell'urea pari a 1 è stato considerato come la dose minima di dialisi da garantire ai pazienti. Una successiva analisi dello studio ha evidenziato come la probabilità di eventi avversi diminuisse in maniera esponenziale all'aumentare del Kt/V dell'urea, suggerendo la possibilità di migliorare l'outcome dei pazienti raggiungendo valori di Kt/V superiori a 1,2 (10).

La risonanza dello studio NCDS fu veramente notevole, e lo dimostra il fatto che i nefrologi progressivamente aumentarono i valori medi di Kt/V dell'urea ottenuti dai pazienti, passando, ad esempio, da 0,99 alla fine degli

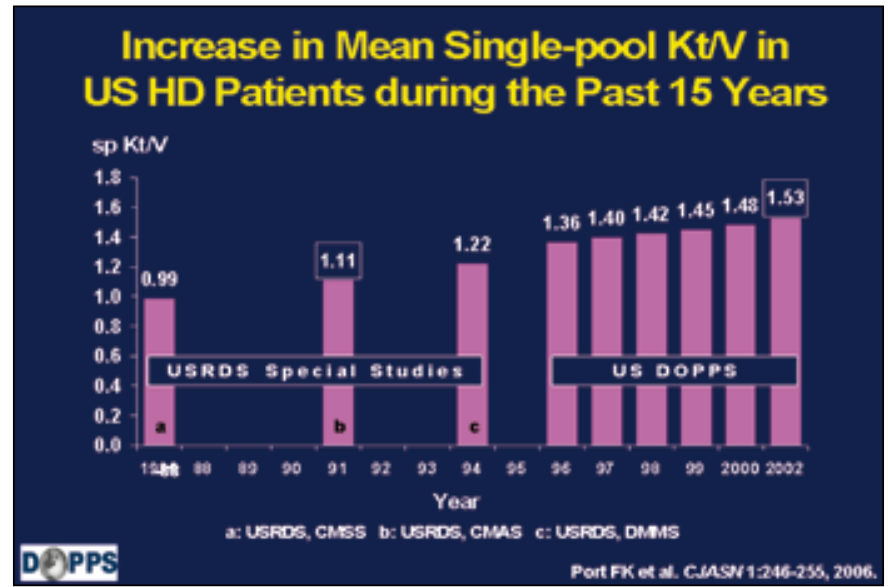

Fig. 1 - Andamento del valore medio di spKt/V nei pazienti emodializzati degli Stati Uniti dalla metà degli anni '80 all'inizio degli anni 2000 (11).

anni Ottanta a 1,53 nel 2002 (Fig. 1), nel tentativo di migliorare la qualità del trattamento sostitutivo e ridurre il tasso di mortalità, che effettivamente migliorarono rispetto al passato, almeno in parte (11).

Nel 1995 iniziava l'HEMO Study, che randomizzava 1846 pazienti prevalenti emodializzati negli Stati Uniti a una dose di dialisi standard vs alta e all'utilizzo di membrane low vs high-flux. Il Kt/V equilibrato dell'urea medio nel gruppo randomizzato a una bassa dose di dialisi era 1,16 ( $\mathrm{spKt} / \mathrm{V}=1,32)$ rispetto a $1,53(\mathrm{spKt} / \mathrm{V}=1,71)$ nel gruppo ad alta dose di dialisi, e i corrispondenti rapporti di riduzione dell'urea (URR, altro parametro utile per valutare la dose di dialisi, meno frequentemente considerato rispetto al Kt/V) erano 66,3 e 75,2. Nel corso di un followup di 2,84 anni, i pazienti assegnati alla dose di dialisi più elevata presentavano una riduzione non statisticamente significativa del $4 \%$ del rischio di morte rispetto a coloro che ricevevano una dose dialitica inferiore, e analoghi risultati emergevano anche dal confronto tra utilizzo di membrane high e low-flux (6).

Attualmente, sulla base dei risultati ottenuti negli studi clinici randomizzati e osservazionali, le Linee Guida K/ DOQI raccomandano di raggiungere un target minimo di spKt/V di 1,2 (12). Nelle Linee Guida della Società Italiana di Nefrologia (SIN) si specifica come la "reale" dose di dialisi sia espressa dal Kt/V all'equilibrio (calcolato con il modello cinetico dell'urea) e si suggerisce un livello minimo accettabile di 1,05 (13), che deve essere aumentato a 1,3 nel caso in cui ci si riferisca al spKt/V. Sin dalla prima fase dello studio DOPPS (DOPPS I) sviluppatasi dal 1996 al 2001 nei Centri dialisi di Francia, Germania, Italia, Spagna, Gran Bretagna, Stati Uniti e Giappone - sono emerse informazioni riguardanti la 
prescrizione emodialitica, e, quindi, anche di dati attinenti la dose di dialisi. $\mathrm{Nel} 2004$, sono stati pubblicati i dati relativi alla depurazione dialitica, raccolti nella popolazione di emodializzati nei cinque Paesi europei partecipanti al DOPPS (Euro-DOPPS), tra Giugno 1998 e Novembre 2000 (14). In particolare, su un campione di 2498 soggetti in emodialisi trisettimanale da più di un anno, sono stati analizzati i parametri della prescrizione dialitica, l'aderenza terapeutica e lo stato nutrizionale. Per quanto riguarda la dose dialitica, veniva valutata sia la dose di dialisi prescritta (calcolata in base al volume di distribuzione dell'urea del paziente, alle caratteristiche del filtro, al flusso ematico e alla durata del trattamento), sia quella somministrata (misurata attraverso il dosaggio dell'urea plasmatica pre- e post-dialisi e l'applicazione della formula di Daugirdas, in modo da quantificare sp e dp Kt/V).

Il primo riscontro degno di nota è stato il fatto che in circa il 25\% dei pazienti in Germania non veniva misurata la dose di dialisi effettivamente somministrata, e questo dimostra come alcune pratiche - benché dimostratesi rilevanti nella pratica clinica quotidiana - non sempre vengano da tutti percepite utili, come invece meriterebbero (14).

I valori medi di dose di dialisi somministrata, sp e dpKt/ $\mathrm{V}$, erano rispettivamente di 1,37 e 1,24, considerando lintera popolazione di soggetti Euro-DOPPS, e quindi al di sopra dei valori minimi consigliati dalle Linee Guida, anche se vi era una considerevole variabilità tra i vari stati, con valori di spKt/V più bassi in Germania $(1,28)$ e più elevati in Francia $(1,50)$. La dose di dialisi somministrata (dpKt/V) era circa 6-19\% inferiore rispetto a quanto prescritto, per motivi che cercheremo di chiarire in seguito (14).

Nel corso degli anni del DOPPS I, è emerso un piccolo - ma statisticamente significativo - declino progressivo della dose di dialisi somministrata, pari a 0,03 punti di spKt/V ogni anno, evidenziabile in tutti gli stati europei partecipanti (Germania in primis con un calo di 0,07 $\mathrm{dpKt} / \mathrm{V} / \mathrm{anno}$ ), con esclusione della Spagna. Tale variazione è stata ritenuta secondaria a un aumento del peso medio dei pazienti emodializzati $(1,8 \mathrm{~kg} /$ anno, variabile tra $0,86 \mathrm{~kg} /$ anno in Italia e $2,79 \mathrm{~kg} /$ anno in Gran Bretagna) e a una riduzione del flusso ematico (calo di $4,4 \mathrm{ml}$ / $\mathrm{min} /$ anno, variabile tra $-1,7 \mathrm{ml} / \mathrm{min} /$ anno in Italia e $-7,4$ $\mathrm{ml} / \mathrm{min} /$ anno in Germania) (14).

Nel DOPPS I, una percentuale rilevante di pazienti presentava un spKt/V inferiore a 1,2 (o dpKt/V inferiore a 1,05$)$, variabile tra il $14 \%$ in Francia e ben il $42 \%$ dei soggetti in Germania. Come rilevato dai dati della fase DOPPS II (Fig. 2), queste percentuali si sono significativamente ridotte, comprese tra il 10\% degli Stati Uniti e il $31 \%$ della Germania (14).

Andando più in dettaglio, una valutazione più estesa e comprendente tutte e tre le fasi dello studio DOPPS,

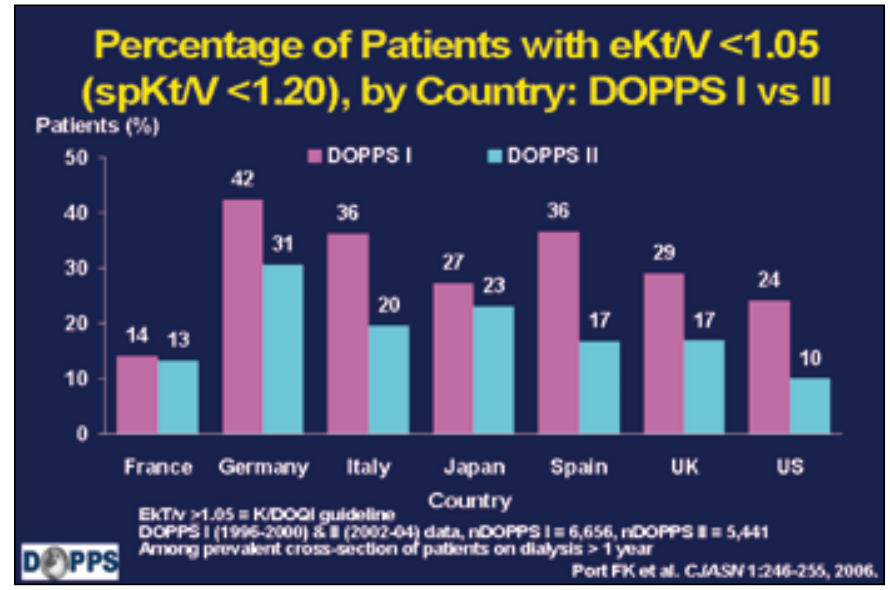

Fig. 2 - Percentuale di pazienti (in dialisi da almeno un anno) con eKt/V < 1,05 (spKt/V <1,20) nel DOPPS I (1996-2000) e II (2002-2004) (11).

mostra come la percentuale di soggetti con spKt/V $<1,2$ si sia sensibilmente ridotta, passando dal $23,1 \%$ rilevato nel 1999 al 18,4\% del 2007. Questo dato, proveniente dalla media di tutti i Paesi DOPPS, è particolarmente rilevante in termini di variazione percentuale in Belgio, Germania, Spagna, Svezia, Stati Uniti e Australia/Nuova Zelanda. In Canada e Giappone si è assistito invece a un lieve aumento dei pazienti con depurazione inadeguata (15). Parallelamente, emerge un cospicuo aumento della quota di pazienti che superano un spKt/V $>1,6$ (dal $24,6 \%$ al 33,6\%) (15).

Anche in Italia sono state registrate tali variazioni nella dose dialitica somministrata, anche se di entità più limitata: nel primo caso (pazienti con spKt/V $<1,2$ ) passando dal 25,3\% al 22,7\% e nel secondo (pazienti con spKt/V $>1,6$ ) dal $26 \%$ al 28,2\%. Mentre, i Paesi più virtuosi si potrebbero considerare Germania - che ha ridotto il numero di soggetti sottodializzati dal pessimo dato di $46,7 \%$ al 22,9\% - e Stati Uniti e Australia/Nuova Zelanda che hanno le percentuali in assoluto minori di spKt/V $<1,2$ (8,7\% e $7,4 \%$ rispettivamente) e maggiori di spKt/V $>1,6$ (48,1\% e $48,5 \%$ rispettivamente) (15).

Lo studio DOPPS ha quindi evidenziato significativi cambiamenti nella pratica clinica quotidiana che, almeno in parte, rispecchiano una maggiore attenzione dei nefrologi nei confronti di quanto raccomandato dalle attuali linee guida nel garantire il raggiungimento di un livello minimo di dose dialitica. Risulta, però, altrettanto evidente anche quanto sia difficile ottenere un'uniformità di comportamento nelle decisioni cliniche, come dimostrato dal numero ancora elevato di soggetti che presentano dati depurativi inadeguati, forse anche per la difficoltà del medico ad accettare il Kt/V come un importante indicatore nella valutazione routinaria dei dializzati (11). 


\section{Raggiungimento dell'adeguatezza dialitica: quali sono i fattori determinanti?}

Diverse componenti concorrono a determinare la dose di dialisi prescritta al paziente: durata della seduta, volume di distribuzione dell'urea del paziente, caratteristiche del filtro, superficie e tipo di membrana, flusso ematico (evidentemente condizionato dalle condizioni dell'accesso vascolare).

Spesso, però, a fronte di una prescrizione dialitica adeguata (in termini di Kt/V teorico ottenibile), la mancata completa applicazione (o applicabilità, più frequentemente) dei parametri prescritti, fa sì che la dose di dialisi somministrata sia di fatto inferiore a quanto desiderato. Lo studio di Hecking ha valutato i vari componenti della prescrizione dialitica, con riscontro di considerevoli variazioni tra i diversi Paesi Euro-DOPPS nel periodo 1998-2000 (14) (Tab. I). Il tempo medio di dialisi era minore in Spagna (216 min), e ben 35 minuti più lungo in Germania (251 min), con una durata media di $234 \mathrm{~min}$, mentre il flusso ematico era compreso tra i $251 \mathrm{ml} / \mathrm{min}$ della Germania e i $322 \mathrm{ml} / \mathrm{min}$ della Spagna $(296 \mathrm{ml} /$ min di media). In Italia, i suddetti parametri erano pari a 221 minuti e $307 \mathrm{ml} / \mathrm{min}$, rispettivamente. Il volume di distribuzione dell'urea medio dei pazienti variava tra 36,4 e 40,7 litri, valori ovviamente correlati anche alle caratteristiche fisiche tipiche della popolazione presa in esame (14).

Per quanto riguarda, invece, le caratteristiche dei filtri di dialisi, quelli con maggiore superficie venivano utilizzati - ancora una volta - in Spagna, con valori ben al di sopra della media Euro-DOPPS (1,72 vs $\left.1,56 \mathrm{~m}^{2}\right)$. In Italia, la superficie media del filtro utilizzata era di $1,50 \mathrm{~m}^{2}$, valore inferiore rispetto alla media suddetta. L'utilizzo di membrane di dialisi sintetiche variava tra il $42 \%$ dei pazienti in Italia e il $71 \%$ in Gran Bretagna, dove vi era il minore utilizzo di membrane high-flux (19\%), che era massimo in Francia (60\%) (14).

I dati del Report DOPPS del 2009 forniscono informazioni dettagliate riguardanti anche l'andamento nel corso delle tre fasi DOPPS del tempo di dialisi e della velocità del flusso ematico prescritti (14).

In merito al tempo di dialisi, dal 1999 al 2007 si è assistito a una generale riduzione del numero di pazienti sottoposti a sedute emodialitiche di durata inferiore a 210 minuti (dal $19,4 \%$ al $12 \%$ ), in favore di un suo progressivo aumento, con un crescente numero di coloro che eseguivano un trattamento di 210-240 minuti o superiore a 4 ore. Benché abbiano registrato un significativo calo nel numero di dializzati per meno di 210 minuti (dal 35,4\% al 24,5\%), gli Stati Uniti sono il Paese DOPPS dove tale

\section{TABELLA I - COMPONENTI DELLA PRESCRIZIONE DIALI- TICA VALUTATE NEI PAESI EURO-DOPPS NEL PERIODO 1998-2000 (14)}

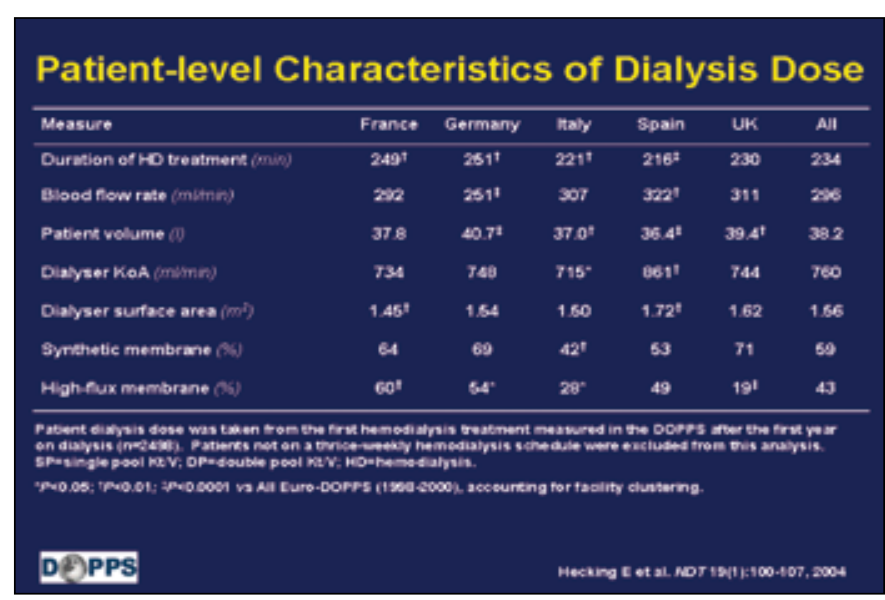

prescrizione temporale è più frequente. In Germania ed Australia/Nuova Zelanda una prescrizione $<210$ minuti è assolutamente rara (intorno al 3\%) e tra il 55 e il $60 \%$ dei pazienti riceve una seduta di durata superiore alle 4 ore (15).

Anche in Italia la durata del trattamento emodialitico è andata incontro a un generale allungamento, come evidenziato dalla riduzione di soggetti trattati per meno di 210 minuti (dal 24,8 al 12,9\%), a favore soprattutto della fascia di durata 210-240 minuti (dal 68,8 al 80\%). Decisamente in controtendenza i dati DOPPS del Giappone, con un aumento delle sedute $<210$ minuti (dal 9,6 al $13,3 \%$ ), una netta diminuzione della fascia $>240$ minuti (dal 21,2 al 15,2\%) e una sostanziale stabilità della durata intermedia (15).

Per quanto riguarda l'entità della velocità del flusso ematico prescritta, i dati disponibili sono più limitati e ristretti al periodo 2002-2007 e non hanno mostrato, in realtà, sostanziali cambiamenti. Informazioni generali, riguardanti gli anni 2006-2007, indicano che nel 50\% circa dei Centri di dialisi si utilizza un flusso ematico compreso tra 250 e $400 \mathrm{ml} / \mathrm{min}$, mentre i rimanenti Centri si distribuiscono equamente verso flussi inferiori $(<250 \mathrm{ml} /$ min) o superiori (>400 $\mathrm{ml} / \mathrm{min}$ ) (15).

Nei vari Paesi DOPPS si possono apprezzare diversi comportamenti. Infatti, in Italia e Francia, la maggioranza dei soggetti ( $90 \%$ circa) si assesta su valori intermedi, mentre vi sono situazioni decisamente opposte, come quanto viene evidenziato in Giappone (oltre il 90\% dei pazienti presenta un flusso ematico inferiore a $250 \mathrm{ml} /$ min) e negli Stati Uniti (oltre l'80\% dei pazienti con un flusso superiore a $400 \mathrm{ml} / \mathrm{min}$ ) (15).

Un'analisi molto interessante evidenziata dal già citato lavoro di Hecking (14) riguardava la relazione tra dose di 
dialisi e categorie di body mass index (BMI). Nella valutazione del rapporto tra $\mathrm{BMI}$ e Kt/V prescritto (spKt/V e dpKt/V) emergeva che i pazienti con BMI più elevato ricevevano la più bassa dose di dialisi. Infatti, nei soggetti con BMI superiore a 25, il Kt/V prescritto, sp e dp era pari a 1,37, 1,29 e 1,17 rispettivamente e decisamente inferiore rispetto ai pazienti con BMI inferiore a 25 . Questi dati europei venivano confermati anche da quelli degli Stati Uniti (16), e sono purtroppo indicativi del fatto che la dose di dialisi viene prescritta dal nefrologo prendendo in considerazione le dimensioni del paziente solo parzialmente, privilegiando invece una prescrizione terapeutica "per durata standard" rispetto a una sviluppata con l'obiettivo di raggiungere una target di Kt/V.

\section{Dose di dialisi e outcome clinico: qual è la relazione?}

Come abbiamo già ricordato, l'HEMO Study è stato realizzato per capire se una dose di dialisi maggiore della media (dpKt/V 1,53 vs 1,16) potesse ridurre la mortalità dei pazienti emodializzati (6). Tuttavia, la riduzione del $4 \%$ del rischio di morte ottenuto nei pazienti randomizzati a ottenere una elevata dose di dialisi non è risultata statisticamente significativa. È però anche possibile il fatto che la riduzione del rischio relativo di morte non sia risultato significativo per l'ampio intervallo di confidenza rilevato, che diventerebbe più stretto all'aumentare del campione di soggetti valutati, come avviene invece negli studi osservazionali, in cui si hanno generalmente a disposizione ampie casistiche di pazienti. Un'analisi secondaria dell'HEMO Study mostrava un beneficio delle membrane high-flux nei pazienti emodializzati da oltre 3,7 anni (6), e nelle donne (ma non negli uomini) (17). Le informazioni provenienti da studi di registro suggeriscono una correlazione non lineare tra $\mathrm{Kt} / \mathrm{V}$ e morbilità o mortalità. Infatti, un aumento del spKt/V medio da 1,1, nel 1991, a 1,3, a metà degli anni Novanta, è stato associato a una riduzione del rischio di morte, decisamente più rilevante rispetto a quella osservata in associazione a un successivo aumento di Kt/V della stessa entità negli anni successivi. Questo indica che un aumento di Kt/V nel range più basso, determinerebbe effetti maggiori rispetto a quanto possa accadere in range di Kt/V più elevati (11). I dati ottenuti dagli studi osservazionali suggeriscono un aumento del rischio di morte con la riduzione della dose di dialisi (18), ed è per questo che le Linee Guida hanno indicato dei livelli minimi di spKt/V. Vi è incertezza quando ci si chiede se una dose di dialisi superiore a quanto suggerito possa decisamente migliorare l'outcome dei nostri pazienti emodializzati.

L'analisi del DOPPS conferma un aumento di mortalità
Relative Risk (RR) of Mortality by Gender and

Dialysis Dose, Measured in eKt/N Dose Categories. based on DOPPS Data

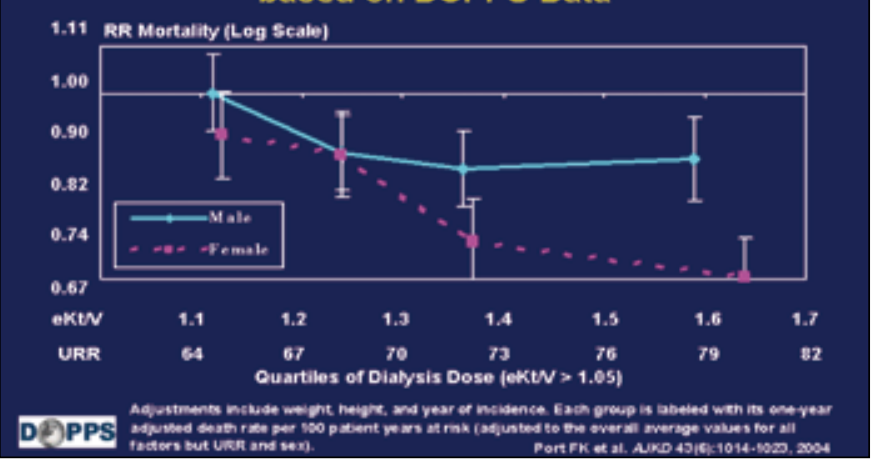

Fig. 3 - Associazione tra rischio relativo di morte e dose di dialisi, valutata separatamente negli uomini e nelle donne (19).

associata a una bassa dose di dialisi (16), e il riscontro dell'HEMO Study di una riduzione della mortalità nelle donne con $\mathrm{Kt} / \mathrm{V}$ più elevato (19).

Infatti, nel 2004, Port e colleghi hanno valutato l'associazione tra dose di dialisi (misurata come URR o dpKt/V) e mortalità - considerata separatamente in uomini e donne - attraverso l'analisi di due numerosi database ("US Centers for Medicare and Medical Services" e DOPPS) (19).

Per quanto riguarda lo studio DOPPS, sono stati presi in considerazione 8438 pazienti in terapia emodialitica da almeno un anno e con un dpKt/V maggiore di 1,05 (spKt/V >1,2). Il rischio relativo di morte, valutato per quartili di dpKt/V, negli uomini e nelle donne (Fig. 3) risultava statisticamente diverso, e in particolare nelle donne si assisteva a una riduzione del rischio relativo di morte pari al $6 \%$ per ogni 0,1 punti di dpKt/V superiore a $1,05(\mathrm{p}<0,001)$, mentre tale percentuale era pari al $2 \%$ negli uomini ( $\mathrm{p}=0,23$, non rilevante dal punto di vista statistico). Inoltre - anche se non confortato da una significatività statistica - emergeva un trend di riduzione di tale effetto positivo per valori di $\mathrm{dpKt} / \mathrm{V}$ superiori a 1,4 (19). I dati DOPPS riscontrati nelle donne sono decisamente sovrapponibili a quanto mostrato dall'HEMO Study.

Alcuni possibili fattori sono stati considerati per spiegare questa osservazione, tra cui il fatto che le donne sembrano essere più complianti alle prescrizioni terapeutiche (compresa l'aderenza all'esecuzione della seduta emodialitica nella sua durata totale) (18), la differenza a livello ormonale legata al sesso e la diversa composizione corporea, in particolare per quanto riguarda la distribuzione del tessuto adiposo (19).

Sempre in tema di possibili rilevanti associazioni tra dose di dialisi e outcomes, nel corso degli ultimi anni sono 
apparse alcune pubblicazioni aventi come base lo stesso tipo di analisi dei dati provenienti dallo studio DOPPS, eseguita però a livello di ognuno dei Paesi partecipanti (20). Nello specifico, veniva considerata la percentuale di emodializzati che non raggiungeva il target raccomandato dalle Linee Guida per sei parametri (tra cui anche la dose di dialisi) che avessero la caratteristica di poter essere modificati attraverso cambiamenti della pratica clinica, associarsi a un'elevata mortalità e per i quali una rilevante percentuale di soggetti presentasse valori al di fuori del range raccomandato. Dopo aver calcolato l'hazard ratio per la mortalità associato ad ogni parametro, in base al numero di pazienti "non in target", è stato calcolato il numero di anni-vita che si potrebbero guadagnare se ogni paziente fosse in grado di raggiungere uno o più target contemporaneamente (partendo dalla premessa che mancato raggiungimento del target e mortalità fossero in un rapporto di causa-effetto) (20).

In Italia, questo studio è stato eseguito su un campione trasversale di 576 pazienti prevalenti della seconda fase del DOPPS (2002-2004) e la percentuale di emodializzati che non conseguivano i target considerati è stata corretta per il numero di pazienti di ogni Centro dialisi, per tenere conto degli squilibri nel campionamento dovuti alle diverse dimensioni dei Centri. I parametri considerati - dose di dialisi, fosforemia, anemia, albuminemia, calcemia e uso di cateteri venosi centrali come accesso vascolare - sono stati confrontati con i target espressi nelle Linee Guida della SIN e le Linee Guida K/DOQI (21). È emerso che, se tutti i pazienti italiani al di fuori dei target raccomandati conseguissero contemporaneamente tutti e sei i target, si potrebbero guadagnare 25.156 anni-vita in cinque anni (o 13.382 anni-vita nel caso in cui solo il $50 \%$ dei pazienti al di fuori dei target li raggiungesse), ottenendo un aumento del 12,7\% del numero di anni-vita attesi per i pazienti emodializzati tra il 2006 e il 2010 (21). Qualora tutti i pazienti potessero ottenere un valore di spKt/V superiore al considerato 1,3 (il $34 \%$ non lo raggiungeva), si potrebbero guadagnare ben 2249 anni-paziente. L'effetto maggiore veniva determinato da albuminemia, seguita dall'uso di cateteri, correzione dell'anemia, fosforemia, dose di dialisi e calcemia (21).

Questo studio pertanto suggerisce la possibilità di migliorare l'aspettativa di vita dei pazienti emodializzati e - sebbene basato su presupposti teorici - i suoi risultati rappresentano sicuramente uno stimolo a perseverare nel continuo processo di miglioramento della pratica clinica attraverso il conseguimento dei target delle Linee Guida, compresa, ovviamente, anche l'adeguatezza della dose di dialisi.

\section{Conclusioni}

Nel corso degli ultimi 30 anni, il Kt/V si è affermato come l'indicatore clinico più comunemente utilizzato nella pratica dialitica quotidiana e si è mostrato in grado di fornire informazioni rilevanti, associate soprattutto all'outcome del paziente. Accanto ai trial randomizzati, anche i dati derivanti dagli studi osservazionali, come lo studio DOPPS, hanno messo in risalto gli aspetti significativi che contribuiscono al raggiungimento dell'adeguatezza dialitica sui quali il nefrologo deve intervenire, perché rappresentano "una valida cartina di torna-sole" della pratica dialitica.

Recentemente, alcuni studi hanno messo in evidenza certi limiti del $\mathrm{Kt} / \mathrm{V}$, conseguenti soprattutto al fatto di normalizzare il prodotto Kt per il volume di distribuzione dell'urea: questa procedura rappresenta un fattore di confondimento, poiché sesso e BMI influenzano di per sé la mortalità del dializzato (22). Tuttavia, l'utilizzo del Kt/V non deve essere abbandonato, ma visto nel più ampio contesto dell'adeguatezza dialitica, in cui esprime il contributo della depurazione delle molecole di basso peso molecolare, che andrà affiancato ai dati riguardanti le molecole di peso molecolare maggiore $(23,24)$. In quest'ottica, il DOPPS, ha più volte sottolineato come possa e debba essere necessario agire anche su altre componenti della prescrizione dialitica, come il flusso ematico, il tempo di dialisi (anche a parità di Kt/V) e l'utilizzo di nuove metodiche dialitiche, come l'emodiafiltrazione on line (11).

\section{Riassunto}

La necessità di misurare oggettivamente la dose di dialisi somministrata ai pazienti ha determinato lo sviluppo di parametri utilizzabili per la valutazione dell'adeguatezza della terapia dialitica, di cui il principale è il Kt/V dell'urea (che mette in relazione la quantità di clearance dell'urea fornita al paziente nel tempo $t$ di trattamento dialitico, con il volume di distribuzione dell'urea).

Due importanti trial randomizzati (il "National Cooperative Dialysis Study" e l'Hemo Study) hanno valutato la dose di dialisi al fine di stabilire il livello minimo da garantire ai pazienti per evitare un aumento di morbilità e mortalità. Ad essi, si sono aggiunti numerosi studi osservazionali che hanno contribuito a definire molti degli aspetti correlati alla dose e all'adeguatezza dialitica. Anche il Dialysis Outcomes and Practice Patterns Study (DOPPS) - studio internazionale, prospettico, osservazionale iniziato nel 1996 per raccogliere dati riguardanti le pratiche cliniche di gestione di problematiche attinen- 
ti all'emodialisi - ha fornito rilevanti informazioni attinenti a tale argomento.

Nel corso degli anni, il DOPPS ha evidenziato una crescente aderenza nei confronti di quanto raccomandato dalle linee guida internazionali, come dimostrato dall'aumento dei valori medi di $\mathrm{Kt} / \mathrm{V}$ e da una percentuale sempre minore di pazienti che presentano una dose di dialisi inadeguata. Inoltre, sono stati raccolti e valutati periodicamente quei parametri della prescrizione dialitica in grado di influire sul raggiungimento del valore di $\mathrm{Kt} / \mathrm{V}$ desiderato e suggerire le possibili modalità di intervento per ottenere i target raccomandati. I dati DOPPS, in accordo con un'analisi secondaria dell'HEMO Study, suggeriscono la possibilità che una più alta dose di dialisi possa associarsi a una miglior sopravvivenza nelle donne.

Pertanto, oggigiorno, il DOPPS rappresenta, anche in fatto di adeguatezza dialitica, un importante riferimento scientifico e un credibile strumento informativo capace di suggerire nuove ipotesi da testare in successivi studi clinici controllati.

Parole chiave: Dose di dialisi, Kt/V, DOPPS, Malattia renale cronica

\section{Indirizzo degli Autori:}

Giuseppe Pontoriero, MD

S.C. Nefrologia e Dialisi

Ospedale "Alessandro Manzoni”

Via dell'Eremo 9/11

23900 Lecco

g.pontoriero@ospedale.lecco.it

\section{Bibliografia}

1. Cavalli A, Del Vecchio L, Manzoni C, Locatelli F. Hemodialysis: yesterday, today and tomorrow. Minerva Urol Nefrol 2010; 62: 1-12.

2. Locatelli F, Buoncristiani U, Canaud B, Kohler H, Petitclerc T, Zucchelli P. Dialysis dose and frequency. Nephrol Dial Transplant 2005; 20: 285-296.

3. De Palma JR, Bolton CF, Baltzan MA, Baltzan RB. Adequate hemodialysis schedule. N Engl J Med 1971; 285: 353-354.

4. Gotch FA, Sargent JA, Keen ML. Individualized, quantified dialysis therapy of uremia. Proc Clin Dial Transpl Forum 1974; 1: 27-37.

5. Lowrie E, Laird N. Cooperative dialysis study. Kidney Int 1983; 23 [Suppl 13]: S1-S122.

6. Eknoyan G, Beck GJ, Cheung AK et al. Effect of dialysis dose and membrane flux in maintenance hemodialysis. N Engl J Med 2002; 347: 2010-2019.

7. Young EW, Goodkin DA, Mapes DL, et al. The Dialysis Outcomes and Practice Patterns Study (DOPPS): an international Hemodialy- sis study. Kidney Int 2000; 57 (Suppl.): S74-S81.

8. Cavalli A, Pontoriero G. Come è cambiata la gestione dell'anemia per i pazienti in dialisi alla luce dello studio DOPPS. Giornale di Tecniche Nefrologiche \& Dialitiche 2010; XXII (4): 27-33.

9. Gotch FA, Sargent JA. A mechanistic analysis of the National Cooperative Dialysis Study (NCDS). Kidney Int 1985; 28: 526-534.

10. Keshaviah P. Urea kinetic and middle molecule approaches to assessing the adequacy of hemodialysis and CAPD. Kidney Int 1993; 43(Suppl 40): S28-S38.

11. Port FK, Pisoni RL, Bommer J et al. Improving outcomes for dialysis patients in the international dialysis outcomes and practice patterns study. Clin J Am Soc Nephrol 2006; 1: 246-255.

12. Levin A, Rocco M, Eknoyan G, et al. Clinical practice guidelines for hemodialysis adequacy, update 2006. Disponibile su: http://www. kidney.org/professionals/kdoqi/guideline_uphd_pd_va/index.htm (accesso 31 maggio 2011).

13. Alloatti S, Basile L, Bosticardo GM, et al. Linee Guida sulla quantificazione della dose dialitica. Disponibile su: http://www.sin-italy.org/pdf/ linee_guida/lg_quant_dose_dialitica.pdf (accesso 31 maggio 2011).

14. Hecking E, Bragg-Gresham JL, Rayner HC, et al. Haemodialysis prescription, adherence and nutritional indicators in five European countries: results from the Dialysis Outcomes and Practice Patterns Study (DOPPS). Nephrol Dial Transplant 2004; 19: 100-107.

15. 2009 Annual Report of the Dialysis Outcomes and Practice Patterns Study: Hemodialysis Data 1999-2008. Arbor Research Collaborative for Health, Ann Arbor, MI._http://www.dopps.org/annualreport/ html/ (accesso 31 maggio 2011).

16. Port FK, Ashby VB, Dhingra RK, Roys E, Wolfe RA. Dialysis dose and body mass index are strongly associated with survival in hemodialysis patients. J Am Soc Nephrol 2002; 13: 1061-1066.

17. Depner T, Daugirdas J, Greene T, et al. Dialysis dose and the effect of gender and body size on outcome in the HEMO study. Kidney Int 2004; 65: 1386-1394.

18. Saran R, Canaud BJ, Depner TA, et al. Dose of dialysis: lessons from major observational studies and clinical trials. Am J Kidney Dis 2004; 44 (Suppl 2): S47-S53.

19. Port FK, Wolfe RA, Hulbert-Shearon TE, McCullough KP, Ashby VB, Held PJ. High dialysis dose is associated with lower mortality among women but not among men. Am J Kidney Dis 2004; 43: 1014-1023.

20. Port FK, Pisoni RL, Bragg-Gresham JL, et al. DOPPS estimates of patient life years attributable to modifiable hemodialysis practices in the United States. Blood Purif 2004; 22: 175-180.

21. Pontoriero G, Locatelli F, Andreucci VE, et al. Stima DOPPS dellimpatto delle linee guida sulla sopravvivenza in emodialisi in Italia. G Ital Nefrol 2007; 3: 221-229.

22. Sarkar SR, Kuhlmann MK, Kotanko P, et al. Metabolic consequences of body size and body composition in hemodialysis patients. Kidney Int 2006; 70 (10): 1832-1839.

23. Jenkins PG. The illogic of Kt/V. Kidney Int 2009; 75 (3): 337.

24. Basile C, Vernaglione L, Lomonte C, et al. Comparison of alternative methods for scaling dialysis dose. Nephrol Dial Transplant 2010; 25 (4): 1232-9. 